\title{
Strongyloides ratti: A Nematode with Extraordinary Plasticity in Aging
}

\author{
Michael P. Gardner, Mark E. Viney and David Gems
}

Aging has been characterized in detail in relatively few animal species. Here we describe the aging process of a nematode with an unusual life-cycle, Strongyloides ratti. This organism has distinct parasitic and free-living reproductive adult forms, which are genetically identical. $S$. ratti exhibits a remarkably high degree of phenotypic plasticity of aging: the maximum lifespan of parasitic adults is 80 -times greater than that of free-living adults (403 and 5 days, respectively). Free-living $S$. ratti adults are short-lived even by terrestrial nematode standards; their lifespan is approximately only $30 \%$ of that of the short-lived free-living nematode model species $C$. elegans. Phenomenologically, aging appears similar in $S$. ratti free-living adults and C. elegans, except that it unfolds much more rapidly in $S$. ratti. Demographic senescence (a hallmark of aging) occurs in free-living $S$. ratti, with a mortality rate doubling time (MRDT) of $0.8 \pm 0.1$ days (females), compared with $2.0 \pm 0.3$ in $C$. elegans. Likewise, parasitic S. ratti undergo senescence, but have an MRDT approximately 30-times greater than that of free-living adults of $S$. ratti. Among nematodes, parasitic species are generally longer-lived than free-living species. This presumably reflects the evolutionary consequences of differences in levels extrinsic mortality experienced in their respective niches. Here, we describe in detail the aging process in the two adult forms of $S$. ratti, and explore how these different lifespans might have evolved, despite sharing the same genome. In this species, an evolved and vast difference in the rate of aging appears to be determined by gene expression alone. This implies that, at least in this one species, "lateacting deleterious mutations" correspond to regulated differences in gene expression that generate a shorter lifespan. Potentially, differences in lifespan between species might evolve via similar mechanisms, involving mutationally driven alterations in gene expression.

\section{Introduction}

Our understanding of aging in certain model species such as the nematode Caenorhabditis elegans, the fruitfly

Handbook for Models of Human Aging

Copyright (C) 2006 by Academic Press

All rights of reproduction in any form reserved.
Drosophila melanogaster, and the mouse is well advanced (Longo and Finch, 2003). However, there are still relatively few animal species for which there are even basic descriptions of how they age. Among nematodes, patterns of aging are highly diverse, and lifespans can vary over more than a 1,000 fold range (Gems, 2001). Strongyloides ratti, the subject of this chapter, is a nematode that has previously been used as a model organism in parasitological studies (Grove, 1989). As a biogerontological model, it is attractive because of its unique level of phenotypic plasticity in aging and the genetics of its lifecycle.

Much has been learned about the genetics of lifespan by the use of mutational studies in model organisms. In C. elegans, increases in lifespan of up to 7-fold have been observed in long-lived mutants (Houthoofd et al., 2004). While substantial, such differences are relatively small, compared with evolved differences in lifespan between different animal species. The genetic basis of such evolved differences remains unknown. It is also unclear whether similar genes and mechanisms underlie both the altered aging in model organisms as uncovered by the analysis of mutants and the evolved differences in aging between different species. These issues may be explored via a comparative approach, and insights gained into the biology of aging from the existing diversity in evolved patterns of aging in the animal kingdom. Thus, it is worthwhile to study the basic phenomenology of aging in species with interesting life histories, such as S. ratti.

Many animal species show phenotypic plasticity in aging, of which there are two major types. The first is the relatively limited response to a changing environment by a given stage in the life-cycle. For example, dietary restriction extends lifespan in many species, including rodents (McCay et al., 1935), Drosophila (Nusbaum and Rose, 1999), and C. elegans (Lakowski and Hekimi, 1998). A more dramatic form of phenotypic plasticity in aging is associated with the development of different morphs within a given life-cycle. For example, in C. elegans there is a facultative diapausal form of the third-stage larva, known as the dauer larva. This longlived stress-resistant dispersal form develops when food is scarce and population density high (Golden and Riddle, 1984) and can survive for up to 3 months (Klass and Hirsh, 1976). This compares to a two-to-three week 
lifespan in C. elegans adults (Klass, 1977). Striking differences in lifespan between adult morphs also occurs in social insects (Finch, 1990), where honey bee queens survive for up to 40-times longer than genetically identical summer worker bees. Understanding the evolutionary and physiological determinants of such plasticity of aging and lifespan can provide insight into the biology of aging.

In this chapter we review what is known about $S$. ratti aging. We describe optimization of culture conditions for aging studies, environmental factors that affect lifespan, and age changes in behavior, pathology and mortality that accompany aging in this unusual nematode. In the concluding section, we consider the question of how the phenotypic plasticity in $S$. ratti may have evolved.

\section{Aging in $S$. ratti \\ WHAT IS S. RATTI?}

Strongyloides is a genus of parasitic nematodes that infect a wide variety of vertebrates (mammals, birds, amphibians, reptiles); two species ( $S$. stercoralis and $S$. fuelleborni) infect humans (Speare, 1989). Some 50 million individuals are infected with Strongyloides spp. worldwide (Grove, 1989). For most individuals there are often no overt signs of infection. However, in individuals that are immunocompromised the infection can disseminate throughout the body, and is fatal in the absence of anti-Strongyloides therapy. S. ratti has been used for some years as a model organism for investigating the biology of nematode parasitic infections (Viney, 1999).

Strongyloides spp. have an unusual and complex lifecycle involving both free-living and parasitic phases (Figure 20.1).

Parasitic adult females dwell in the gut of the host (which in the case of S. ratti, as its name implies, is the rat). Here they burrow into the intestinal mucosa, and reproduce by mitotic parthenogenesis (Viney, 1994), passing eggs via the feces into the external environment. These eggs can either moult through two larval stages into infective third stage larvae (iL3s) or through four larval stages into free-living adult females and males (Figure 20.1). These adults mate by conventional sexual reproduction and produce progeny which (as above) can develop into iL3s (Viney et al., 1993; Harvey et al., 2000). Infective L3s infect new hosts by skin penetration. Experimental genetic studies found that reproduction of the parasitic females of $S$. ratti is functionally mitotic (Viney, 1994) and so the progeny of the parasitic female are genetically identical to each other and to their mother. Anecdotal observations had noted a large difference in the lifespan potential of the adult free-living and parasitic female morphs: approximately one week and one year, respectively (Viney, 1994; Gemill et al., 1997).

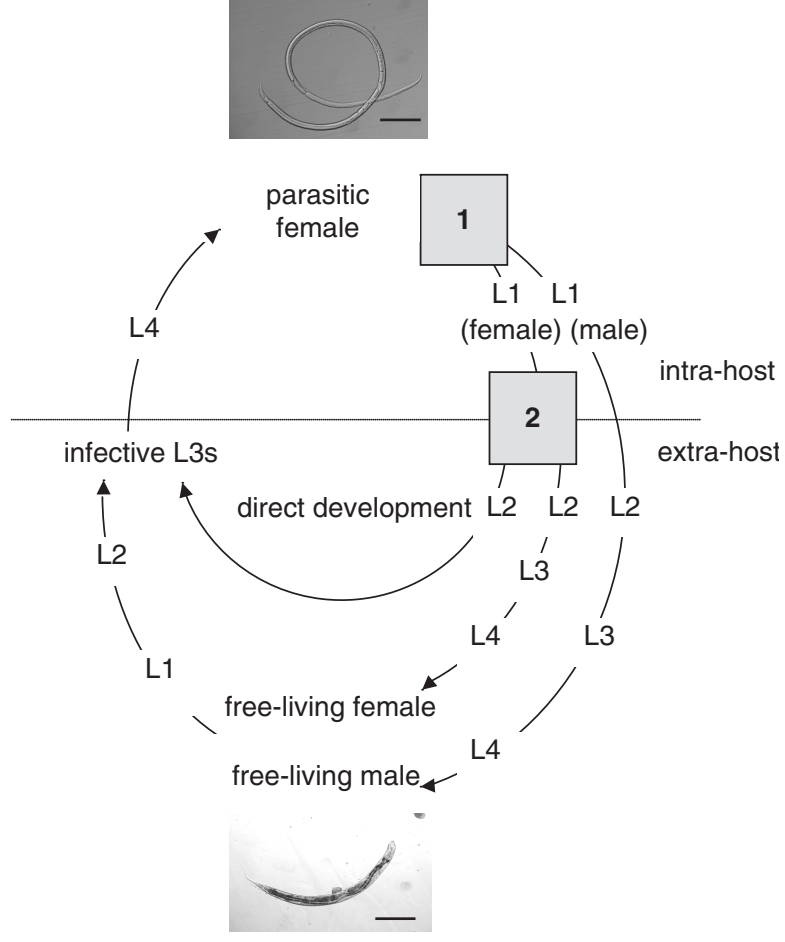

Figure 20.1. Life-cycle of Strongyloides ratti with two discrete developmental switches, shown as grey boxes: (1) a sex determination event; (2) a female-only developmental switch. Larval stages are omitted for clarity. Adapted from Harvey et al., (2000). Also included are larval stages L1-L4. The life-cycle contains both parasitic and free-living generations; an adult parasitic female (left) and free-living adult female (right). Bars $=200 \mu \mathrm{m}$.

However, the role of aging in this apparent difference was unknown.

\section{CULTURE CONDITIONS FOR S. RATTI}

\section{Parasitic S. ratti}

The parasitic stages of $S$. ratti are maintained in the laboratory by infecting rats. Immunologically normal animals mount an immune response against these infections, such that the infections are lost after four to six weeks (Wilkes et al., 2004). However, long-term infections can be established in immunologically deficient (nude) rats (Gemill et al., 1997). Feces collected from infected rats contain the eggs or first-stage larvae. These feces can be collected and used to make fecal cultures, from which all the free-living stages of the life-cycle can be grown. Infective L3s obtained from such cultures are used to initiate new infections, which is done by the subcutaneous administration of iL3s to naïve rats (Gemill et al., 1997; Wilkes et al., 2004).

Free-living S. ratti

For the analysis of aging and lifespan, the culture conditions used for the free-living adult S. ratti are 


\section{Strongyloides ratti: A Nematode with Extraordinary Plasticity in Aging}

based on those used for C. elegans. The latter are typically maintained on NGM agar seeded with Escherichia coli strain OP50 (a uracil auxotroph) as a food source (Sulston and Hodgkin, 1988). For S. ratti, L4 female and male worms are taken from 2-day old rat fecal cultures $\left(19^{\circ} \mathrm{C}\right)$, cleaned by repeated washing in sterile distilled water to remove associated bacteria, and then introduced onto NGM plates under optimized culture conditions (see below). To measure lifespan, survival of the free-living stages of $S$. ratti is measured at least daily, with scoring for death as described for C. elegans (Klass, 1977).

Cultures of $C$. elegans are monoxenic, that is, only the $E$. coli food source is present. In contrast, S. ratti larval stages derived from fecal cultures are inevitably contaminated with fecal bacteria, which may reduce their
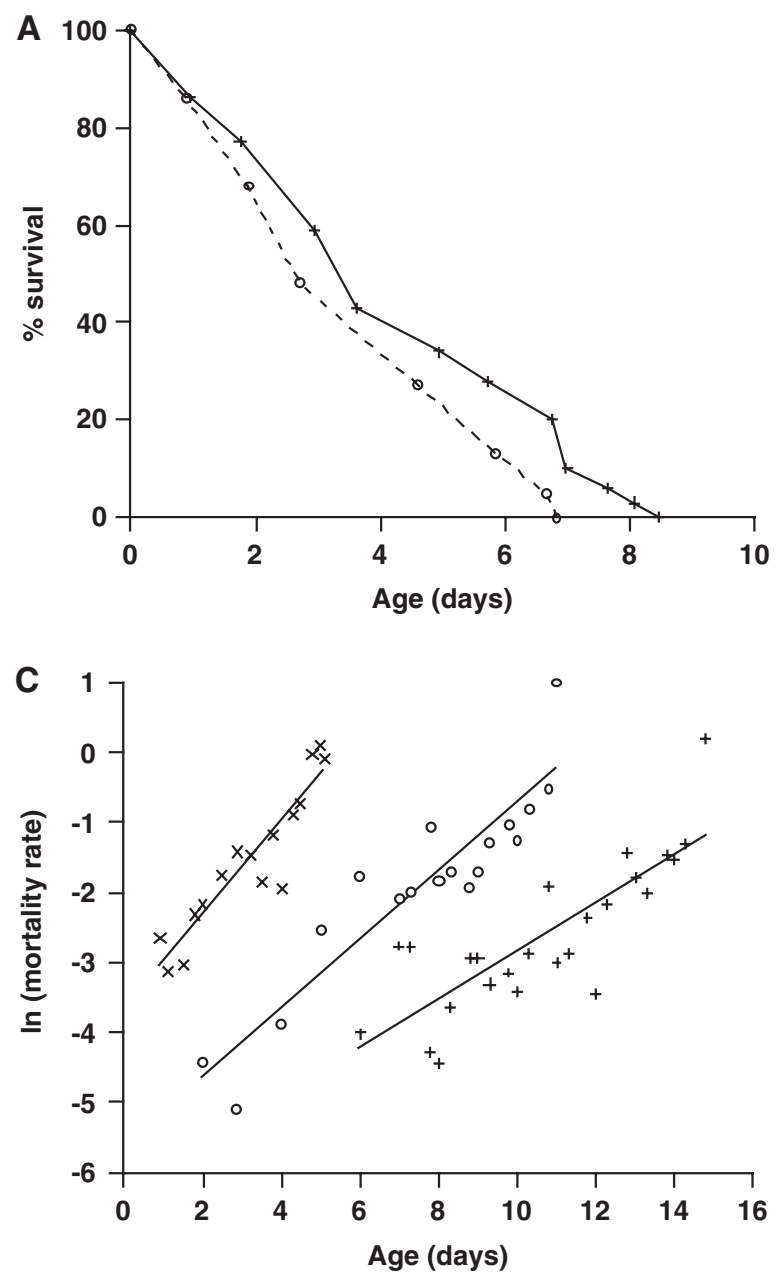

lifespan. To minimize this problem, the agar plates on which these $S$. ratti free-living stages are cultured contain $400 \mu \mathrm{g} / \mathrm{ml}$ streptomycin and are seeded with a streptomycin-resistant (SR) E. coli OP50 food source. Under these conditions, visible contamination by other microbes is reduced, but not prevented. With these conditions the maximum mean lifespan ( $\pm \mathrm{SE}$ ) of $S$. ratti females was $4.2 \pm 0.2$ days. This lifespan is significantly greater than that of $S$. ratti maintained without the presence of streptomycin (Gardner et al., 2004; Figure 20.2A).

\section{MEASURING AGING IN S. RATTI}

Analysis of age-specific mortality

One feature of aging that has been observed throughout the animal kingdom is the exponential acceleration of
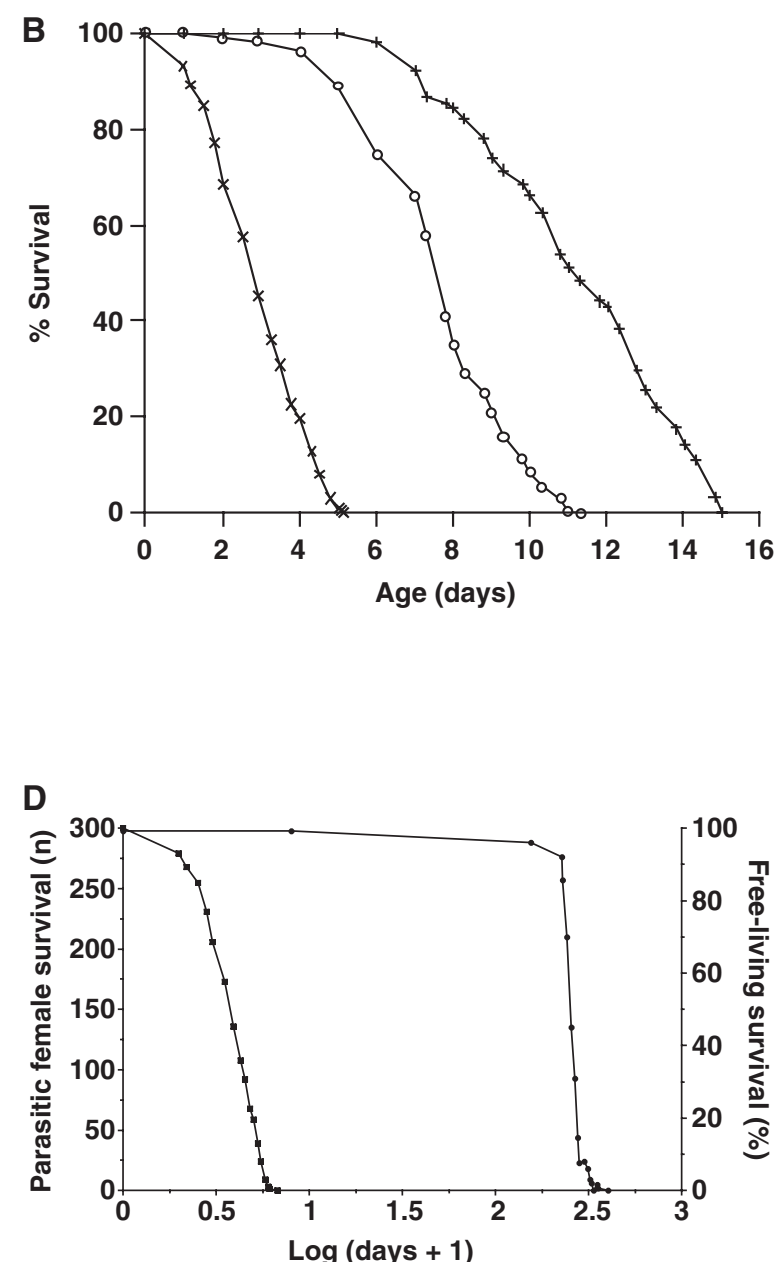

Figure 20.2. Factors affecting $S$. ratti lifespan. (A) Survivorship of virgin free-living females under optimized culture conditions $(400 \mathrm{mg} / \mu \mathrm{l}$ streptomycin and E. coli SR) $(\mathrm{x}, \longrightarrow$ ) and on E. coli OP50 (o, - . -). (B) Survival and (C) Plots of $\ln (m(t))$ against age for $S$. ratti $(x, \longrightarrow)$ and C. elegans $(0,-)$ both in the S, ratti-optimized culture conditions $(400 \mathrm{mg} / \mu \mathrm{l}$ streptomycin and E. coli SR) and C. elegans $(+, \longrightarrow)$ in monoxenic culture conditions E. coli OP50. (D) The estimated number of parasitic females (•) and the percent survival of free-living females (-) over time, which is shown on a log scale. Figures 20.2D, 20.3C, and 20.4C are from Gardner, Gems and Viney, submitted. Figures 20.1, 20.2A-C, 20.3A-B and 20.4A-B are reprinted from Gardner, Gems and Viney, Aging in a very short-lived nematode, Experimental Gerontology Volume 39, 1267-1276 (2004) with permission from Elsevier. 


\section{Michael P. Gardner, Mark E. Viney and David Gems}

the mortality rate with increasing age (Finch, 1990) The pattern of age-specific mortality in many species (including humans) can be described by the Gompertz equation, $m(t)=A e^{\alpha t}$, where $m(t)$ is the mortality rate at time $t, A$ is the initial mortality rate (IMR) and $\alpha$ is the Gompertz exponential function (Finch, 1990). Population cohorts may be extinguished as the result of mortality that is related or unrelated to aging; therefore, differences in lifespan per se do not necessarily reflect differences in the rate of aging. Limitations of lifespan by aging, as opposed to some other extrinsic cause of mortality (e.g. predation or disease), may be established by testing for the occurrence of an exponential increase in mortality rate with increasing age (Wachter and Finch, 1997). From plots of $\ln$ (mortality rate) against age, the IMR and the Gompertz exponential function can be calculated. The latter may be expressed in a form that is easier to grasp: the mortality rate doubling time (MRDT). These mortality parameters for free-living $S$. ratti females and parasitic $S$. ratti females are described below.

Age-specific mortality in S. ratti free-living adult females

The survival of free-living $S$. ratti females under optimized $S$. ratti culture conditions are shown in Figure 20.2B. This shows that its maximum lifespan is $4.5 \pm 0.8$ days. In our studies of free-living $S$. ratti we were at pains to distinguish whether the very short lifespan resulted from rapid aging, or from some form of pathology distinct from aging. To this end, we compared age changes in $S$. ratti with those in $C$. elegans. The latter was cultured either monoxenically (i.e. with E. coli OP50 alone), or under the same conditions as $S$. ratti (i.e. with SR OP50 and low level contamination with other microbes). (Figure 20.2B).

The mean lifespan of $C$. elegans hermaphrodites under either culture condition $(S$. ratti conditions: $7.7 \pm 0.1$ days; monoxenic: $11.2 \pm 0.1$ days) was substantially and significantly longer than that of $S$. ratti $(3.0 \pm 0.1$ days $)$ (Gardner et al., 2004; Figure 20.2B). Unsurprisingly, $C$. elegans lifespan was greater in monoxenic culture conditions than in $S$. ratti optimized culture conditions, presumably due to the presence of contaminating microbes in the S. ratti culture conditions (Figure 20.2B).

In all cases, an age-specific mortality rate acceleration was observed (Figure 20.2C), consistent with the occurence of senescence (i.e. intrinsic aging). For $S$. ratti the MRDT $( \pm$ SE) was lower and the IMR $( \pm$ SE) higher $\left(0.8 \pm 0.1\right.$ days; $0.025 \pm 0.002$ days $^{-1}$, respectively) than in C. elegans (in either culture condition). For C. elegans under the two culture conditions, there was no difference in the MRDT ( $S$. ratti-conditions: $1.4 \pm 0.2$ days; monoxenic conditions: $2.0 \pm 0.3$ days), but the IMR for $C$. elegans kept under $S$. ratti culture conditions was almost twice that of monoxenically cultured populations ( $S$. ratti conditions: $0.0036 \pm 0.0003$ days $^{-1}$; monoxenic: $0.0018 \pm 0.0002$ days $\left.^{-1}\right)$. Overall, these findings imply that $S$. ratti is shorter lived than C. elegans because (a) it is more frail, as reflected by higher IMR, and (b) it ages more quickly, as reflected by a lower MRDT.

Age-specific mortality in $\mathrm{S}$. ratti parasitic females In $S$. ratti parasitic females, maximum lifespan is 403 days (Gardner, Gems and Viney, submitted; Figure 20.2D), which compares to 5 days in the free-living morph (Figure 20.2D).

The maximum lifespan of $S$. ratti parasitic females is 403 days, which is some 80 -times greater than the 5-day lifespan of the free-living females. The parasitic females underwent an exponential increase in mortality rate with age, thereby showing that senescence was occuring. The IMR of the parasitic females was 0.0056 days $^{-1}$, which is approximately $25 \%$ of that of the free-living females. The MRDT of the parasitic females was 22.7 days, which is some 30-times longer than that of the free-living females. Overall, this comparison of the parasitic and free-living females implies that freeliving morphs are shorter-lived due to their increased frailty (as reflected by the greater IMR) and a faster rate of aging (as reflected by lower MRDT). $S$. ratti is the first parasitic nematode in which the occurrence of demographic senescence has been demonstrated in the laboratory.

\section{FACTORS AFFECTING LIFESPAN IN FREE-LIVING S. RATTI AND C. ELEGANS}

The lifespan of the free-living adult female $S$. ratti is really very short; in fact it is the shortest-lived nematode reported. One possibility is that this very short lifespan is not the result of aging in the usual sense. For example, mortality might result from failure to feed, or a gross anatomical defect not seen in other nematodes. To address these issues we compared aging in free-living $S$. ratti with that in C. elegans in two ways. Firstly, we tested the effect of factors known to affect aging in C. elegans on aging in $S$. ratti free-living adults. Secondly, we asked whether the age-associated changes in C. elegans behavior, morphology and appearance are also seen in $S$. ratti. Overall, our findings strongly support the view that the short lifespan in $S$. ratti free-living adults is the result of typical nematode aging, but which is happening at an unusually swift pace.

In C. elegans, lifespan is reduced by various factors, including the E. coli food source (Gems and Riddle, 2000; Garigan et al., 2002), higher temperature (Klass, 1977), mating between the sexes (Gems and Riddle, 1996) and attempted mating between males (Gems and Riddle, 2000). In S. ratti, lifespan (Figure 20.3A) and lifetime fecundity (Figure 20.3B) were reduced by higher temperatures, as in C. elegans (Klass, 1977).

However, mating did not affect lifespan in S. ratti in the same way as it does in C. elegans. Thus, mating (manipulated by adjusting the male:female ratio from 0 to 4.0) had no effect on $S$. ratti free-living female lifespan, 

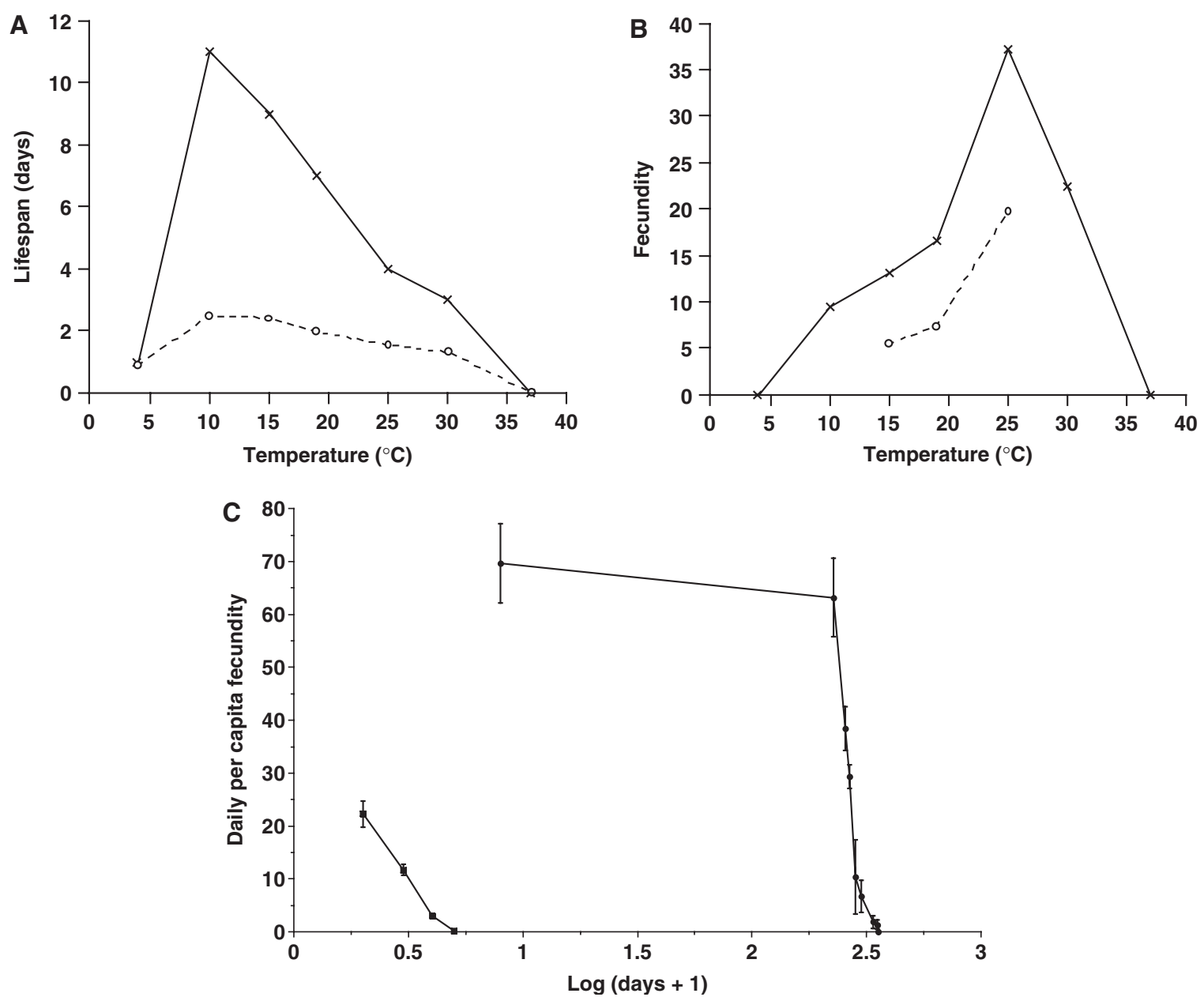

Figure 20.3. (A) Effects of temperature on mean $\left(\mathrm{O}, \ldots\right.$ - - ) and maximum $\left(x_{,}-\right.$) lifespan of virgin free-living females. S. ratti adults die within 24 hours at $37^{\circ} \mathrm{C}$. (B) Effect of temperature on mean per capita lifetime fecundity of S. ratti females, mated on day one only $(0, \ldots-)$ or mated throughout life $\left(x_{1}-\right)$. (C) The mean $( \pm 1 \mathrm{SE})$ daily per capita fecundity of the parasitic females $(-)$ and of the free-living females ( $\mathbf{n}$ ) (mean $\pm 1 \mathrm{SE}$; adapted from Gardner et al., 2004) over time, which is shown on a log scale.

whereas in males it actually increased lifespan. Thus lifespan in $S$. ratti and C. elegans are affected in the same way by some factors (temperature, food source (data not shown), proliferating bacteria) but in different ways by others (mating).

\section{BEHAVIORAL CHANGES ACCOMPANYING AGING IN S. RATTI}

Fecundity

The daily per capita fecundity of free-living S. ratti females fell rapidly with age, as in C.elegans, but more rapidly, halving between the first and second days of adult life (Figure 20.3C). The resultant maximum lifetime fecundity was less than 40 progeny per female, compared to over 1,400 in C. elegans (Hodgkin and Barnes, 1991). By contrast, the daily per capita fecundity of the parasitic adult morph was $>50$ for $c$. 250 days before it declined, giving a lifetime fecundity of $\sim 16,000$ (Gardner, Gems and Viney, submitted; Figure 20.3C).

Pharyngeal pumping rate and movement

In C. elegans, the rate of pharyngeal pumping (reflecting the rate of ingestion of food) declines gradually with age (Kenyon et al., 1993). We observed a similar agerelated decline in pharyngeal pumping rate in $S$. ratti. We also saw a similar age-related decline in movement in S. ratti, as in C. elegans (Herndon et al., 2002). In each case the pattern of age-related change in $S$. ratti was similar, but more rapid than that in C. elegans.

\section{MORPHOLOGICAL CHANGES ACCOMPANYING AGING IN S. RATTI}

\section{Autofluorescence}

In $C$. elegans, there is an increase in intestinal autofluorescence with age (Garigan et al., 2002), similar to 
accumulation of age-pigment (lipofuscin) seen during mammalian aging. There is a similar increase in autofluorescence with age in free-living $S$. ratti morphs (Gardner et al., 2004). By contrast, little such autofluorescence was seen in parasitic females, even in 11-month-old individuals (Gardner, Gems and Viney, submitted). Lipofuscin accumulation reflects organismal failure to detoxify and excrete molecular waste products. The paucity of autofluorescence in $S$. ratti parasitic females suggests reduced generation, or/and increased excretion, of damaged molecular constituents. This is consistent with aging occurring in the two $S$. ratti female morphs, but at very different rates.

Microscopic examination of aging nematodes

The integrity of major anatomical features was compared in young and old S. ratti adults using differential interference contrast (DIC) and transmission electron microscopy (TEM) (Gardner et al., 2004; Gardner, Gems and Viney, submitted). Overall, the appearance of aging $S$. ratti free-living females was similar to that of aging C. elegans (Herndon et al., 2002). The pharynxes of older free-living $S$. ratti were frequently distended and blocked with bacteria. Such pharyngeal blockage has also been observed in older C. elegans (Garigan et al., 2002). The most striking morphological age changes in free-living $S$. ratti occurred in the intestine (Gardner et al., 2004). Whilst in one-day-old animals there were large, healthy looking refractile intestinal cells (Figure 20.4A), in the majority of four-day-old animals these cells were severely atrophied and the cytoplasm had a ragged appearance (Figure 20.4B).

By contrast, older parasitic females showed no marked degenerate changes.

TEM observations of free-living females showed numerous inclusions in the intestinal cytoplasm whose
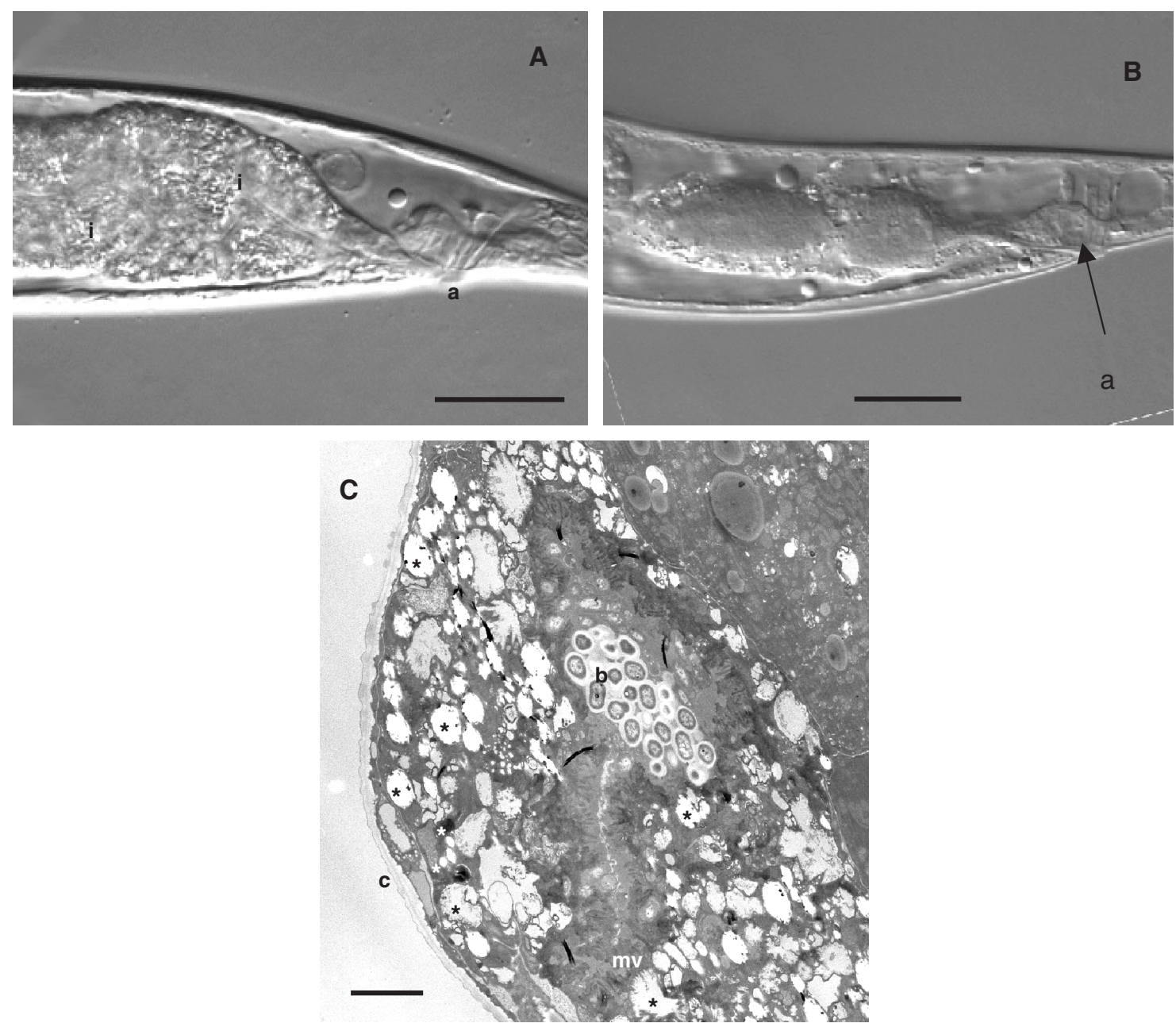

Figure 20.4. (A-B) S. ratti free-living females viewed using differential interference contrast (Nomarski) microscopy. (A): one-day-old female; (B), four-day-old adults. (A) and (B): posterior intestine. In (A), note the large, healthy refractile intestinal cells (i). In (B), the intestine is strikingly atrophied. Anus (a). Bars $=20 \mu \mathrm{m}$. (C) TEM of four-day-old free-living females with electron-dense inclusions of intestinal cells indicated by * (Bar $=2 \mu \mathrm{m})$; c, cuticle; mv, microvilli; $b$, clump of bacteria in lumen.

\author{
AU: Please \\ check units. \\ Abbreviation \\ for "meter" = m, \\ not $M$.
}


number increased with age (Figure 20.4C). Most of these resembled lipid droplets (or possibly secondary lysosomes) within which have accumulated electronopaque particles with a sooty appearance. By contrast, there were very few such inclusions in the intestines of parasitic females and their number did not change with age. These observations suggest that degeneration of the intestine, possibly associated with accumulation of fluorescent/electron opaque waste material, represents a pathology of aging that contributes to nematode pathology. This idea was previously put forward based on TEM studies of aging in Caenorhabditis briggsae (Epstein et al., 1972). The fact that this pathology was not detected in the intestine of the parasitic $S$. ratti adult is consistent with idea that the absence of this pathology contributes to parasitic longevity.

The parasitic and free-living females morphs of $S$. ratti are genetically identical. Therefore differences between these morphs must be due to differences in gene expression. This being so, these differences in intestinal pathology might reflect differences in regulated biochemical processes which determine longevity and aging. One intriguing and attractive possibility is that in parasitic females there is an increase in biochemical processes promoting detoxification. Recent microarray studies of long-lived insulin/IGF-1 signalling mutants of C. elegans have implicated a broad range of detoxification processes in longevity assurance (Murphy et al., 2003; McElwee et al., 2004; Gems and McElwee, 2005). These include phase 1 and phase 2 drug detoxification, as well as antioxidant enzyme and chaperonin activities. Most of these processes require energy input, and excretion of solublized toxic metabolites by the drug detoxification system occurs via the smooth endoplasmic reticulum. Interestingly, intestinal cells of parasitic $S$. ratti adults are rich with mitochondria and smooth endoplasmic reticulum.

\section{Conclusion}

\section{LEARNING ABOUT LIFESPAN EVOLUTION FROM S. RATTI}

In this chapter we have described the uniquely high level of phenotypic plasticity in aging in Strongyloides ratti, corresponding to an 80 -fold difference in lifespan, accompanied by a 400 -fold difference in maximum fecundity. While these represent something of a comparative gerontological party trick, does it provide any real insight into the biology of aging? Arguably, the existence of plasticity of this sort has implications for the genetic mechanisms underlying lifespan evolution.

Evolutionary theory predicts that organisms in environments with a low rate of extrinsic mortality will evolve slower rates of aging than those in environments with a high rate of extrinsic mortality (Medawar, 1952; Williams, 1957). This is because high levels of extrinsic mortality reduce selection against alleles which produce deleterious effects later in life. As a consequence, populations experiencing higher extrinsic mortality will accumulate such late-acting deleterious alleles, which cause aging. If alleles are pleiotropic, and capable of producing phenotypic effects at different time in an animals life, there may be selection for alleles which enhance fitness due to early effects, despite later deleterious effects (antagonistic pleiotropy).

The relationship between extrinsic mortality rate and aging has been supported by numerous comparative studies. For example, rodents generally live longer than bats of a similar size, presumably since flight aids predator evasion, reducing extrinsic mortality (Wilkinson and South, 2002). Evolution results in intraspecific as well as interspecific differences in lifespan. For example, in social insect species there are intercaste differences in lifespan which may reflect the evolutionary effects of different levels of extrinsic mortality on rates of aging (Page and Peng, 2001; Chapuisat and Keller, 2002). However, the extent to which these lifespan differences are the result of differences in the rate of aging remains unclear. For example, Chapuisat and Keller (2002) compared survival in large and small worker ants, and found that the smaller workers lived significantly longer, which they hypothesized was because the larger morphs were fighting and getting killed more quickly.

Thus here lifespan differences are not due to differences in aging; rather, just differences in extrinsic mortality rates.

Although the evolutionary theory of aging provides an explanation for how different aging rates evolve, in terms of molecular or developmental genetics, the concept of "late-acting deleterious mutations" remains frustratingly abstract. Potentially, the pattern of aging in $S$. ratti provides a clue as to the molecular genetic basis of lifespan evolution, as follows. Free-living nematodes are typically much shorter lived than parasitic nematodes, some of which have lifespans of over a decade (Gems, 2001). These evolved differences are likely to reflect differences in extrinsic mortality experienced by free-living versus parasitic species. Hence the difference in lifespan of free-living and parasitic S. ratti seems consistent with the evolutionary theory of aging. What is interesting about $S$. ratti is that the two adult forms have evolved such a vast difference in aging rate, despite the fact that they are the same species, sharing a common genome.

That this is possible sheds some light on the nature of "late-acting deleterious mutations." According to an evolutionary interpretation, free-living $S$. ratti have evolved a short lifespan due to high extrinsic mortality rate, and accumulation of alleles with deleterious late acting effects. However, one might expect that these alleles would be purged from the population by selection against them in the parasitic adult. The nature of $S$. ratti clearly demonstrates that this has not happened, and in this sense its evolution represents a useful experiment

\section{AU: Therefore shouldn't bats live longer than rodents? Please clarify.}




\section{Michael P. Gardner, Mark E. Viney and David Gems}

of nature. What is also clear from $S$. ratti is that the evolved difference in lifespan between the two adult forms is the product of differential gene expression. This tells us that, at least in this one species, "late-acting deleterious mutations" actually reflect regulated differences in gene expression leading to shorter lifespan.

Aging is a trait which shows remarkable evolutionary plasticity, and is able to evolve rapidly. For example, humans and chimpanzees have evolved a difference in maximum lifespan of some 50 years since divergence from a common ancestor only 6-7 million years ago. It has been suggested that there are "lifespan regulatory modules," which are regulated sets of genes which control the rate of aging (Kenyon, 2005). The existence of these makes possible the observed rapid rates of evolutionary change in aging rates. Some clues to the possible nature of the genes involved in lifespan are emerging from new genetic studies of aging in model organisms.

\section{BETWEEN THE GENETICS AND EVOLUTION OF LIFESPAN}

Major advances have recently been made in terms of the genetics of lifespan, particularly using $C$. elegans and $D$. melanogaster. For example, the insulin/insulin-like growth factor (IGF-1) signalling pathway has been shown to be a powerful regulator of lifespan in nematodes, flies and probably rodents (Kenyon, 2005). The relationship between the lifespan-determining genes and pathways identified, and those involved in lifespan evolution remain unclear. One possibility is that at least some of the genes and processes identified by model organism lifespan genetics are the same as those involved in lifespan evolution. In the case of $S$. ratti, insulin/IGF-1 signalling is a good candidate for a regulator of phenotypic plasticity of aging. In $C$. elegans, this pathway controls the differences in lifespan between the longlived dauer larva, and the shorter lived adult (Riddle and Albert, 1997).

In C. elegans, DAF-16, a FOXO-family transcription factor, controls the rate of aging in response to insulin/ IGF-1 signalling. Recently, microarray studies have begun to identify the transcriptional targets of DAF-16, which are predicted to include the ultimate genetic determinants of longevity and aging in $C$. elegans (Murphy et al., 2003; McElwee et al., 2004). DAF-16 alters the expression of a wide-range of genes, involved in many life processes, including defences against stress, toxins and microbial invasion. To date, these analyses of the role of IIS and DAF-16 have been based on the analysis of mutants, where lifespan differences of up to 2.5 fold can be achieved (Kenyon et al., 1993). While substantial, such differences are much less than evolved, interspecific differences in lifespan, or the 80-fold differences in lifespan between the different adult morphs of S. ratti. Therefore, a plausible hypothesis is that $S$. ratti lifespan plasticity is determined by differential expression of the same sorts of genes and processes regulated by DAF-16 in C. elegans. Further, one may speculate that this is also the case for lifespan differences between species, such as that between human and chimpanzee. A graphic representation of this hypothesis is presented in Figure 20.5.

Overall, this discussion of the implications of $S$. ratti aging suggests a view of lifespan as a highly plastic, regulated trait that is consistent with the rapid evolution of interspecific differences in lifespan.

\section{WHY ARE FREE-LIVING S. RATTI SO VERY SHORT LIVED?}

One remaining oddity of $S$. ratti is that the free-living adults are really very short-lived, even by the standards of short-lived free-living nematode species. There are several possible explanations for this. Firstly, the freeliving adult phase of the life-cycle is facultative (Viney, 1996). Hence when it does not occur, this may weaken selection on lifespan in the same manner as high extrinsic mortality. Secondly, it might result from antagonistic pleiotropy between the effects of genes on fitness in the free-living and parasitic forms. Aging may reflect action of alleles that increase early-life fitness (e.g. by increasing reproductive output) but have deleterious late-life effects (Williams, 1957). Such antagonistic pleiotropy is supported by experimental investigation (Kirkwood and Austad, 2000; Partridge and Gems, 2002). Hence, in the case of $S$. ratti, the shortevity of the free-living adults may result from the greater fecundity of the parasitic female, which may favor pleiotropic alleles that increase parasitic fecundity but reduce lifespan of free-living adults.

\section{LEARNING ABOUT AGING FROM COMPARATIVE STUDIES}

Studies concentrating on a small number of model organisms have figured strongly in recent biogerontological

Figure 20.5. A hypothetical scheme for the mechanism of lifespan regulation and evolution. (A) Evolved intraspecific differences in lifespan between parasitic and free-living adults are generated by differences in gene expression, regulated by endocrine and cellular signalling systems via regulated transcription factors. We propose that, as in C. elegans (Murphy et al., 2003), parasitic adult longevity in S. ratti results from up-regulation of longevity assurance genes and down-regulation of genes which promote aging. $\mathrm{A}$ arrows $=$ stimulation, and $\mathrm{T}$ bars $=$ inhibition. (B) Potentially, differences in aging rate between species may evolve via similar mechanisms. Here, the environment of an ancestral species changes, such that the extrinsic mortality rate is lowered, and selection against deleterious late life effects is increased. This leads to the evolution of slower aging and increased longevity, which might occur via alterations in regulation of longevity assurance and aging genes. This predicts that several types of gene are likely to be altered during lifespan evolution: those encoding endocrine, signalling proteins and transcription factors. In (B), asterisks represent alterations in the trait resulting from random mutation, and selection. 
A

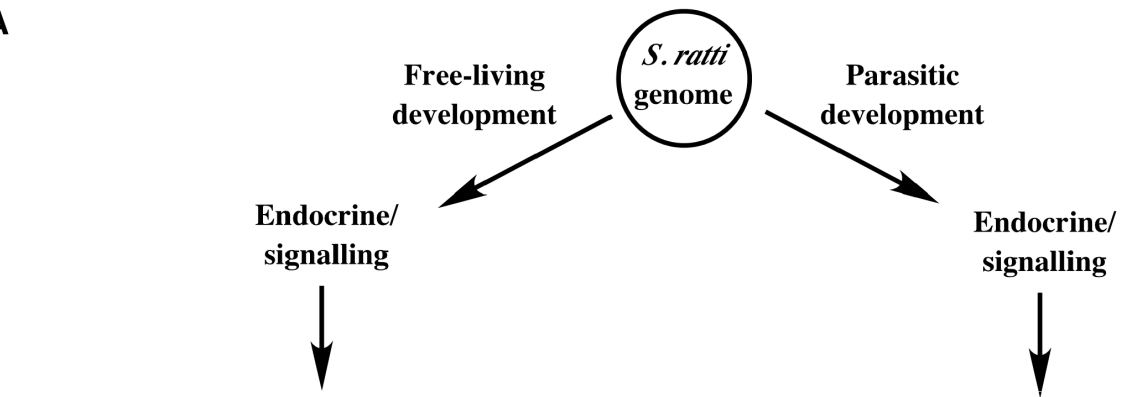

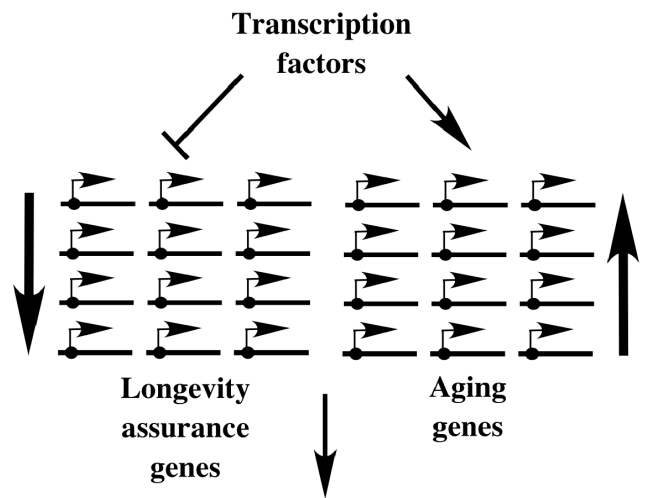

Short-lived

free-living

adult

B

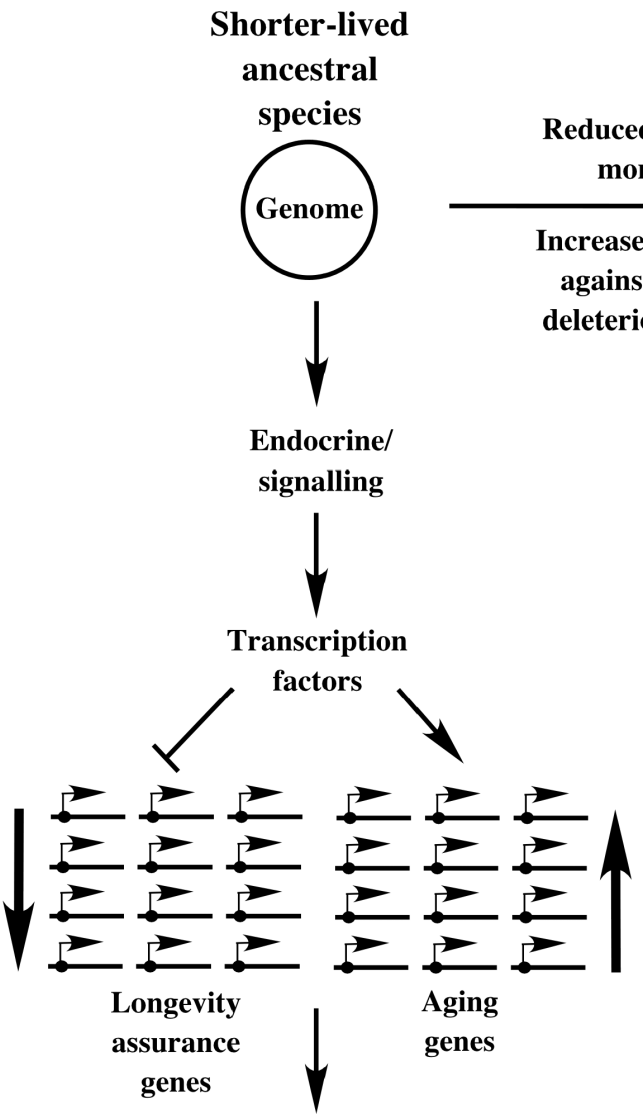

Shorter

life span

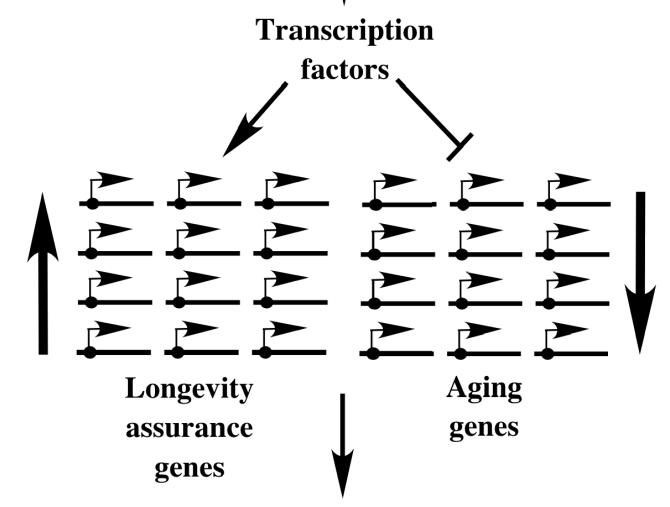

Long-lived parasitic adult

Longer-lived

descendant

species

Genome

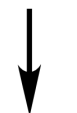

Endocrine/ signalling*

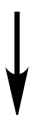

Transcription

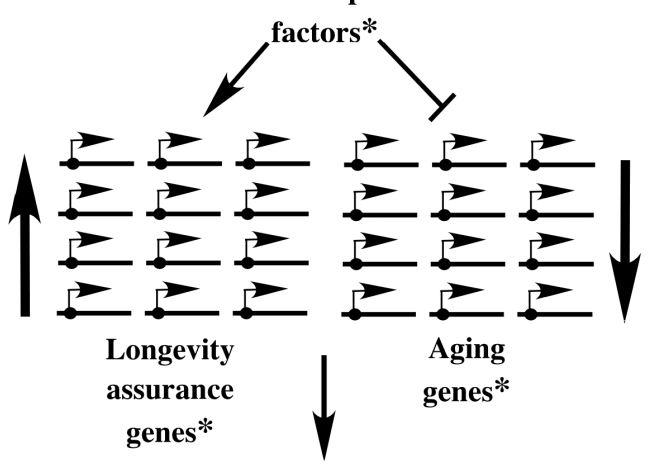

Longevity 
research. While this approach has been very fruitful indeed, studies of $S$. ratti aging illustrate the value of limited investigations of the biology of aging in selected animal species. Such studies in the comparative biology of aging can yield fundamental facts about the biology of aging. A good example of this is the study of Hydra vulgaris which revealed an apparent absence of senescence in this species (Martinez, 1998). This demonstrates that aging is not a necessary feature of adult metazoan biology. It seems likely that there are many unusual and informative patterns of aging to be discovered, particularly among lower invertebrates, which may include the absence of senescence, facultative nonsenescence and examples of phenotypic plasticity of aging even more extreme than in $S$. ratti. The possible value of organisms with exotic patterns of aging is likely to increase as the ease of genome sequencing increases and its expense falls. Comparative functional genomic approaches, using whole genome microarray analysis, applied to such organisms, has the potential to yield insights into both the biology of lifespan determination, and the evolution of aging and longevity.

\section{Recommended Resources}

Below are listed useful Web sites and suggested further reading (books and review articles).

\section{S. RATTI REVIEWS/PAPERS}

Aging has been characterized in detail in relatively few animal species. In his review article and book chapter below, David Gems underlines the potential importance of comparative studies of aging in nematodes.

Gems, D., 2001. Longevity and aging in parasitic and free-living nematodes. Biogerontology 1, 289-307.

Gems, D., 2002. Ageing. In: Lee, L.D. (Ed.). The Biology of Nematodes. Taylor and Francis, London and New York, pp. 413-455.

In the study below, we described the aging process in free-living adults of the parasitic nematode Strongyloides ratti.

Gardner, M.P., Gems, D., and Viney, M.E., 2004. Aging in a very short-lived nematode. Exp. Gerontol. 39, 1267-1276.

Gardner, M.P., Gems, D., and Viney, M.E. 2005. Extraordinary plasticity of ageing in a nematode. Submitted.

\section{S. RATTI/PARASITIC NEMATODE WEBSITES}

ESTs of S. ratti http://www.nematode.net/Species. Summaries/Strongyloides.ratti/index.php

Genome sequence of parasitic nematode B. malayi http://www.tigr.org/tdb/e2k1/bma1/

\section{GENERAL AGING BOOKS}

Finch, C.E., 1990. Longevity, Senescence and the Genome, University of Chicago Press, Chicago.

\section{ACKNOWLEDGMENTS}

MPG was supported by a grant to MEV and DG from the Experimental Research on Ageing Programme of the BBSRC and we thank them for their support. MEV is supported by the MRC, NERC and the Wellcome Trust. DG is also supported by funds from the EU (Framework V), the Wellcome Trust, and the Royal Society.

\section{REFERENCES}

Chapuisat, M., and Keller, L., 2002. Division of labour influences the rate of ageing in weaver ant workers. Proc. R. Soc. London Ser. B 269, 909-913.

Epstein, J., Himmelhoch, S., and Gershon, D., 1972. Studies on aging in nematodes III. Electronmicroscopical studies on ageassociated cellular damage. Mech. Ageing Dev. 1, 245-255.

Finch, C.E., 1990. Longevity, Senescence and the Genome, University of Chicago Press, Chicago.

Gardner, M.P., Gems, D., and Viney, M.E., 2004. Aging in a very short-lived nematode. Exp. Gerontol. 39, 1267-1276 (2004).

Garigan, D., Hsu, A -L., Fraser, A.G., Kamath, R.S., Ahringer, J., and Kenyon, C., 2002. Genetic analysis of tissue aging in Caenorhabditis elegans: A role for heat-shock factor and bacterial proliferation. Genetics 161, 1101-1112.

Gemmill, A.W., Viney, M.E., and Read, A.F., 1997. Host immune status determines sexuality in a parasitic nematode. Evolution 51, 393-401.

Gems, D., 2001. Longevity and ageing in parasitic and free-living nematodes. Biogerontology 1, 289-307.

Gems, D., and McElwee, J.J., 2005. Broad spectrum detoxification: The major longevity assurance process regulated by insulin/IGF-1 signalling? Mech. Ageing Dev. 126, 381-387.

Gems, D., and Riddle, D.L., 1996. Longevity in Caenorhabditis elegans reduced by mating but not gamete production. Nature 379, 723-725.

Gems, D., and Riddle, D.L., 2000. Genetic, behavioural and environmental determinants of male longevity in Caenorhabditis elegans. Genetics 154, 1597-1610.

Golden, J.W., and Riddle, D.L., 1984. The Caenorhabditis elegans dauer larva: Developmental effects of pheromone, food and temperature. Dev. Biol. 102, 368-378.

Grove, D.I., 1989. Historical introduction. In Grove, D.I. (Ed.). Strongyloidiasis: A Major Roundworm Infection of Man. Taylor and Francis, London and New York, pp. 1-9.

Harvey, S.C., Gemill, A.W., Read, A.F., and Viney, M.E., 2000. The control of morph development in the parasitic nematode Strongyloides ratti. Proc. R. Soc. London Ser. B 267, 2057-2063.

Herndon, L.A., Schmeissner, P.J., Dudaronek, J.M., Brown, P.A., Listner, K.M., Sakano, Y., et al., 2002. Stochastic and genetic factors influence tissue-specific decline in ageing C. elegans. Nature 419, 808-814.

Hodgkin, J., and Barnes, T.M., 1991. More is not better: brood size and population growth in a self-fertilizing nematode. Proc. R. Soc. London Ser. B 246, 19-24.

Houthoofd, K., Braeckman, B.P., Johnson, T.E., and Vanfleteren, J.R., 2004. Extending life-span in C. elegans. Science. 305, 1238-1239.

\section{CE: Journal style varies from other chapters. Followed MSS. Please verify.}




\section{Strongyloides ratti: A Nematode with Extraordinary Plasticity in Aging}

Kenyon, C., 2005. The plasticity of aging: Insights from long-lived mutants. Cell 120, 449-460.

Kenyon, C., Chang, J., Gensch, E., Rudener, A., and Tabtiang, R.A., 1993. A C. elegans mutant that lives twice as long as wild type. Nature 366, 461-464.

Kirkwood, T.B.L., and Austad, S.N., 2000. Why do we age? Nature 408, 233-238.

Klass, M.R., 1977. Aging in the nematode Caenorhabditis elegans: Major biological and environmental factors influencing life span. Mech. Ageing Dev. 6, 413-429.

Klass, M.R., and Hirsh, D.I., 1976. Non-ageing development variant of Caenorhabditis elegans. Nature 260, 523-525.

Lakowski, B., and Hekimi, S., 1998. The genetics of caloric restriction in Caenorhabditis elegans. Proc. Natl. Acad. Sci. USA 95, 13091-13096.

Longo, V.D., and Finch, C.E., 2003. Evolutionary medicine: From dwarf model systems to healthy centenarians? Science 299, 1342-1346.

Martínez, D.E., 1998. Mortality patterns suggest lack of senescence in hydra. Exp. Gerontol. 33, 217-225.

McCay, C., Crowell, and M., Maynard, L., 1935. The effect of retarded growth upon the length of the life span and upon the ultimate body size. J. Nutr. 10, 63-79.

McElwee, J.J., Schuster, E., Blanc, E., Thomas, J.H, and Gems, D., 2004. Shared transcriptional signature in C. elegans dauer larvae and long-lived daf-2 mutants implicates detoxification system in longevity assurance. J. Biol. Chem. 279, 44533-44543.

Medawar, P.B., 1952. An Unsolved Problem of Biology. H.K. Lewis, London.

Murphy, C.T., McCarroll, S.A., Bargmann, C.I., et al., 2003. Genes that act downstream of DAF-16 to influence the lifespan of Caenorhabditis elegans. Nature 424, 277-284.

Nusbaum, T., and Rose, M., 1999. The effects of nutritional manipulation and laboratory selection on lifespan in Drosophila melanogaster. J. Gerontol. A Biol. Sci. Med. Sci. 54, B192-B198.
Page, R.E., and Peng, C.Y.-S., 2001. Aging and development in social insects with emphasis on the honey bee, Apis mellifera L. Exp. Gerontol. 36, 695-711.

Partridge, L., and Gems, D., 2002. Mechanisms of ageing: Public or private? Nat. Rev. Genet. 3, 165-175.

Riddle, D.L., and Albert, P.S., 1997. Genetic and environmental regulation of dauer larva development. In C. elegans II (eds. D.L. Riddle, T. Blumenthal, B.J. Meyer, J.R. Priess), pp. 739-768. Cold Spring Harbor Laboratory Press, New York.

Speare, R., 1989. Identification of species of Strongyloides. In: Grove, D.I. (Ed.). Strongyloidiasis: A Major Roundworm Infection of Man. Taylor and Francis, London and New York, pp. 11-83.

Sulston, J., and Hodgkin, J., 1988. Methods. In: Wood, W.B. (Ed.). The Nematode Caenorhabditis elegans. Cold Spring Harbor Press, New York, pp. 587-606.

Viney, M.E., 1994. A genetic analysis of reproduction in Strongyloides ratti. Parasitology 109, 511-515.

Viney, M.E., 1996. Developmental switching in the parasitic nematode Strongyloides ratti. Proc. R. Soc. London Ser. B 263, 201-208.

Viney, M.E., 1999. Exploiting the life-cycle of Strongyloides ratti. Parasitology Today 15, 231-235.

Viney, M.E., Matthews, B.E., and Walliker, D., 1993. Mating in the nematode parasite Strongyloides ratti: Proof of genetic exchange. Proc. R. Soc. London Ser. B 254, 213-219.

Wachter, K.W., and Finch, C.E., 1997. Between Zeus and the Salmon, National Academy Press, Washington DC.

Wilkes, C.P., Thompson, F.J., Gardner, M.P., Paterson, S., Viney, M.E., 2004. The effect of the host immune response on the parasitic nematode Strongyloides ratti. Parasitology 128, 661-669.

Wilkinson, G., and South, J., 2002. Life history, ecology and longevity in bats. Aging Cell 1, 124-131.

Williams, G.C., 1957. Pleiotropy, natural selection and the evolution of senescence. Evolution 11, 398-411. 
File: $\{$ Elsevier\}Conn/Pageproofs/3d/P369391-Ch20.3d

Creator: abdu//CIPL-U1-REV-03.unitt.cepha.net Date/Time: 24.10.2005/5:06pm Page: 240/240 